\title{
PWiseHA: Application of Harmony Search Algorithm for Test Suites Generation using Pairwise Techniques
}

\author{
Aminu Aminu Muazu ${ }^{1}$ \\ Department of Computer Science \\ Umaru Musa Yar'aduaUniversity \\ Katsina, Nigeria. \\ Email:aminu.aminu [AT] umyu.edu.ng
}

\author{
Umar Danjuma Maiwada ${ }^{2}$ \\ Department of Computer Science \\ Umaru Musa Yar'adua University \\ Katsina, Nigeria. \\ Email: umar.danjuma [AT] umyu.edu.ng
}

\begin{abstract}
Pairwise testing is an approach that tests every possible combinations of values of parameters. In this approach, number of all combinations are selected to ensure all possible pairs of parameter values are included in the final test suite. Gene rating test cases is the most active re search area in pairwise testing, but the generation process of the efficient test suite with minimum size can be conside red as one of optimization problem. In this research paper we articulate the problem of finding a pairwise final test suite as a search problem and the application of harmony search algorithm to solve it. Also, in this research paper, we developed a pairwise software testing tool called PWise HA that will generate test cases using harmony search algorithm and this PWiseHA is well optimized. Finally, the result obtained from $P W$ iseHA shows a competitive results if matched with the result of existing pairwise testing tools. PWise HA is still in prototype form, an obvious starting point for future work.
\end{abstract}

Keywords--- Software testing, Pairwise testing, interaction strength, Test suites, Harmony search algorithms.

\section{INTRODUCTION}

The entire aspiration of a software company is to ensure that a software is delivered with a high quality to it customers [1] [2]. Therefore, to achieve a high-quality software, the software need to be tested. The software testing makes sure that software achieves the user requirements, such that to avoid failures visible to customers. Testing is very important phases in software development lifecycle. Lack of testing may lead to harmful consequences which include the loss of an important data, the fortunes, and even the lives of people [3]. The main aim of software testing is to minimize the recognized software fault which is not accepted [4] [5] [6]. As such, the software engineers need only to consider a significant huge number of test data. Software testing refer to sequence of processes that was aimed to ensure that the software configuration does what it was planned to do and that it does not do something unplanned [7]. To test all likely combinations of inputs data and execution paths is called exhaustive testing, but it's beyond our reach [8].

Combinatorial testing refer to a specification based testing standard that requires for each $d$-way (where $d$ indicates the combination strength degree) combination of input parameters of a given system, for every combination of a valid values of these $d$ parameters can becovered by at least one test case [7] [9]. In pertinent literature, it is reported that $d$-way interaction small value of $d$ is more effective in testing. Combinatorial testing is communal as a real technique to uncover accidental feature interactions which are confidential to a given software system. This tenacity has made clear that test cases are created by mingling tuples that are from different input parameters. This approach was recognized with success in terms of providing a very low cost testing in our true situations. The critical is sues of tes ting a software is to detect software faults and generate an optimized test suite. Combinatorial testing provides probability for fault detection, which triggered by the interaction among parameters in the software under testing [10]. For this large interaction space, the exhaustive testing is generally impractical, even though there are av ailable resources (took) to do it, because most of the interaction values do not cause any failure. Combinatorial testing provides the smaller test suite that cover the large interaction parameter values [11] [12].

Combinatorial testing is an organized approach for sampling large provinces of test data. Observations have been found that most of the system faults are encountered when there is interactions between parameters values [7] [11]. This is the origin of pairwise definition, which can also refer to as 2 -wise testing. In this techniques number of all combinations are selected set by set to ensure all possible pairs of parameter values are included in the final test suite. On the otherhand, many evidences suggest that most software failures are caused by an unwanted pairwise interactions between the parameters of a system[13]. 
Only one is sues is considered most in pairwise testing, that is, each pairwise interaction most be covered by at least one test in the final test suite [14].

Most of the time, testing all the test cases (exhau stively) is always impossible, this is because of timing cons traints and als o resources [15] [16]. The main problemis

In this research paper, we expressed the problem of generating pairwise test suites as a search problem and applying harmony search algorithm in solving the problem Also, we introduced a pairwise tool called PWiseHA that may well serve as a configuration for generating pairwise test suites with harmony search algorithm.

We carry out a successions of experiments in order to evaluate the effectiveness and performance of our pairwise prototype tool normally known as PWiseHA. In lieu of the evaluation, we have us ed the available benchmark problems from the pairwise site [17]; it has presented list of many tools for generating pairwise test suits, where most of them included with their competence actions in terms of generating final test suites. Our tactic is more reasonable in that, if related with the existing pairwise tools in [17]. PW iseHA is still in a prototype form, which is an obvious starting point for future work would be to complete the implementation.

\section{PROBLEM STATEMENT}

Nowadays software systems can be run with different number of configurations, these configurations are made up of parameters and their respective values. All of these configurations need to be considered during testing.

The pairwise type of testing is a combinatorial testing skill that tests all possible pairs of input parameter values [18]. The most leading challenge in pairwise testing is that to find a test suites which consist of the smallest number of test cases that covers all pairs of input parameters of a software system [19]. Normally, in pairwise testing the capable way of finding a best solution is not predictable, because the time needed to generate the test cases grows promptly as the increased numbers of parameters with their respective values. Although, there are many existing pairwise strategies that minimized the number of test cases in a software system, but most of these strategies are not well optimized; rather they provide an acceptable solutions [20].

The exact research problem is therefore to develop a harmony search algorithmprototype tool which is proficient for creating and reducing as much as promising test suites that contain all pairs of input parameters values of the software under testing. The basics behind choosing the harmony search algorithm in PWiseHA is that it has the power to control the search between the local solutions and global solutions based on its parameters [20]. to reduce the quantity of combinations while keeping the effectiveness of detecting errors. Some number of techniques have been explored such that to address this particular problem. Therefore, pairwise is a better technique to prevent many of this problems.

\section{RELATED WORK}

Software testing consumes most of the time and cost spent on software development. Basically, a lot of researchers developed different pairwise strategies to solve this problem and by generating an optimized tes t suite [4] [5].

The Automatic Efficient Test Generator (AETG) is a tes ting tool that constructs a test suite by adopting one test at a time approach using greedy algorithm [21]. The InParameter Order (IPO) is a testing tool strategy that constructs a test suite by adopting one parameter at a time using a horizontal and vertical algorithm [1] [12]. The Test Configuration for pairwise interaction (TConfig) is a testing tool that constructs a test suite using recursive algorithm [17]. The main idea for Jenny strategy is it start with one pair (itself) and it will then search for if there is other one pair, if there is not then it goes to two pairs. It will also search for if there are other 2-pairs, if not it will then go to 3-pairs. The same thing for other pairs. Therefore, the process will continue until all pairs are covered [17]. The Disability Discrimination Act (DDA) constructs a test suite using greedy algorithm [17]. The All-Pairs Testing (AllPairs) is a tes ting tool that constructs a test suite using greedy algorithm [17]. The Pairwise Independent Combinatorial Tool (PICT) constructs a test suite using core generation alg orithm with randomselection [17], PICT is not reliable to provide a nonoptimal test size when compared with other strategies because of randomconduct [14]. The rest: the Combinatorial Test Services (CTS) tool, the TestCover testing tool, the EXACT testing tool, the IPO family known as IPOS, the ecFeed testing tool, and the JCUnit testing tool uses some sort of determinis tic algorithms [19].

\section{MATERIALS ANDMETHODOLOGY}

\section{A. Harmony Search Algorithms for painwise testing}

The Harmony search first begun when listening to an attractive piece of standard music. This is when musicians compose the harmony, they frequently try many possible combinations of the music pitches that are stored in the memory, which can be considered as an optimization process of adjusting the pitches (input) to obtain the optimal output (a perfect harmony). Harmony search draws the inspiration from harmony improvisation and has gained good result in the optimization area [4] [22]. 


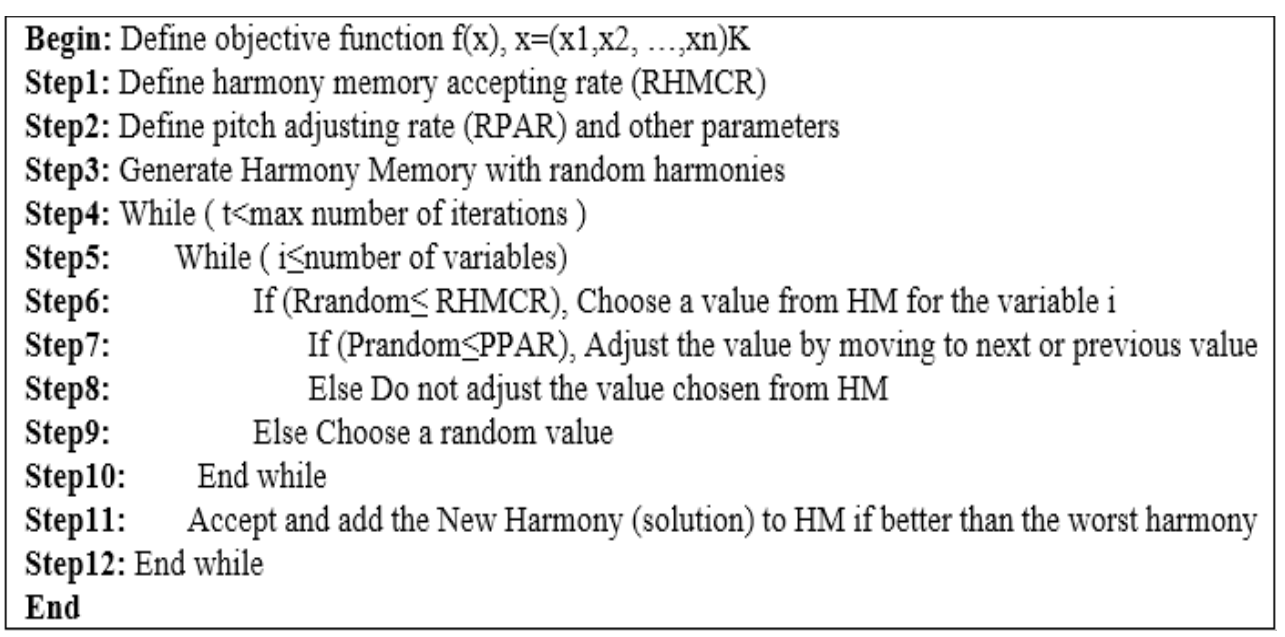

Figure 1. Pseudocode of Harmony search algorithm [14].

The music improvisation is simply a way for searching a better harmony, and it's achieved when attempting different combinations of pitches that satis fies the following rules [23]:

- Playing any one pitch from the memory

- Playing an adjacent pitch of one pitch from the memory

- Playing a randompitch from the possible range

This method is represented in each variable selection of the Harmony Search algorithm. Similarly, it should follow any of the three rules below:

○ Choosing any value from the Harmony Search memory

- Choosing an adjacent value fromthe Harmony Search memory

- Choosing a random value from the possible value range

The above mentioned three rules in the harmony search algorithm are maintain by the following parameters: Harmony Memory Considering Rate(HMCR) and Pitch Adjustment Rate (PAR) [22] [24].

The first step will initialize the harmony memory. Now the harmony memory consists of a number of randomly generated solutions to the optimization problem under consideration, whereby these test cases are generated using pairwise techniques.

The second step will improvise a new solution from the harmony memory. Each component of this solution is obtained based on the HMCR. Still here the test cases were generated using pairwise techniques.

The third step will update the harmony memory. Also new test pair which was generated from the second step is evaluated and if it's better than the worst in the harmony memory, it will replace it. Else, it will eliminated.
The fourth step will repeat second s tep to third stepuntil a present termination criterion is met (i.e. the maximal number of iterations is met).

Harmony Search algorithmis in used successfully in an extensive variety of optimization problems. It present numerous advantages with respect to traditional optimization techniques [23]. Figure 1 displays the pseudocode of Harmony Search Algorithm.

\section{B. PWiseHA Implementation}

This prototype tool contain only one algorithm that will optimize and generate a near optimal final test suite. Here in this algorithm the concept and procedures in harmony search is applied that would work with pairwise tes ting techniques. The steps used in PW is eHA are as follows:

$1^{\text {st }}$ Step: Here it will initialize the harmony search memory. $2^{\text {nd }}$ Step: Here it will improvise a new solution from the hamony memory.

$3^{\text {rd }}$ Step: Here it will update the harmony memory.

$4^{\text {th }}$ Step: Here it will repeat second step to third step until a present termination criterion is met.

In Figure 2, we have displays the pseudocode of PWiseHA strategy. This PWiseHA prototype tool was developed using Java programming language (in JCreator LE 4.50 environment) and Java found ation classes AWT and Swing are used for the Graphical User Interface (GUI) with JDK 1.6. Here, the Java programming language has been chosen because it has the features of platform-independent (which means it has the ability to run the same program on different operating systems), java GUI is user friendly, and also it has rich API's for manipulation of array lists. 







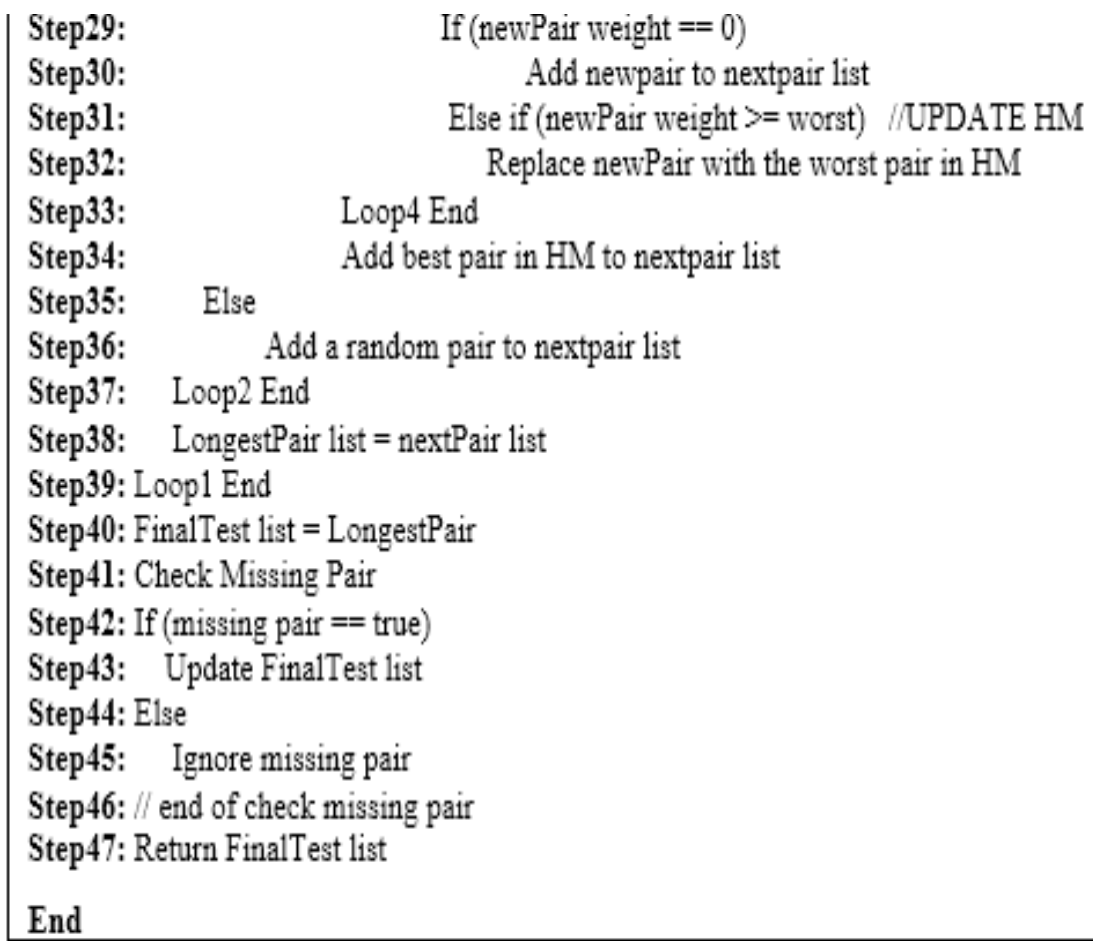

Figure 2. Pseudocode of PWiseHA

The PW is eHA prototype tool s tructure contains a set of GUI, a set of java clas ses, a simple database to store the final test suit result and Operating System(OS). All the components of
PWis eHA strategy depends on the OS layer, which will run the overall prototype. Some fragment codes of PWiseHA were shown in Figure 3.

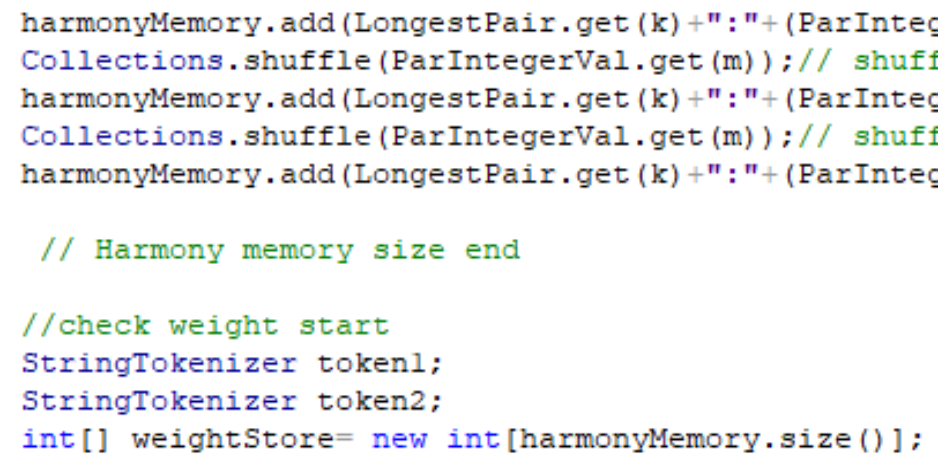

Figure 3. Some fragment codes of PWiseHA

The PW iseHA GUI's were developed in such away it has many attributes which requires a test engineer to read and execute the configuration test file and displays the final test suit. This attribute are illus trated in the TABLEI.

TABLE I. THE PWISEHA FORM ATTRIBUTES

\begin{tabular}{lll}
\hline Attribute Name & \multicolumn{1}{c}{ Type } & \multicolumn{1}{c}{ Description } \\
\hline Read input file & Button & It will read a text file \\
\hline Generate test case & Button & It will generate the final test suite \\
\hline Clear & Button & It will clear all the text on text area \\
\hline Exit & Button & It will close the window \\
\hline & Text Area & It will display a result \\
\hline
\end{tabular}


The Figure 4 present the PW is eHA form with a displayed information of final test suite. Finally, the implementation of
PWiseHA prototype tool has been demonstrated in fine points.

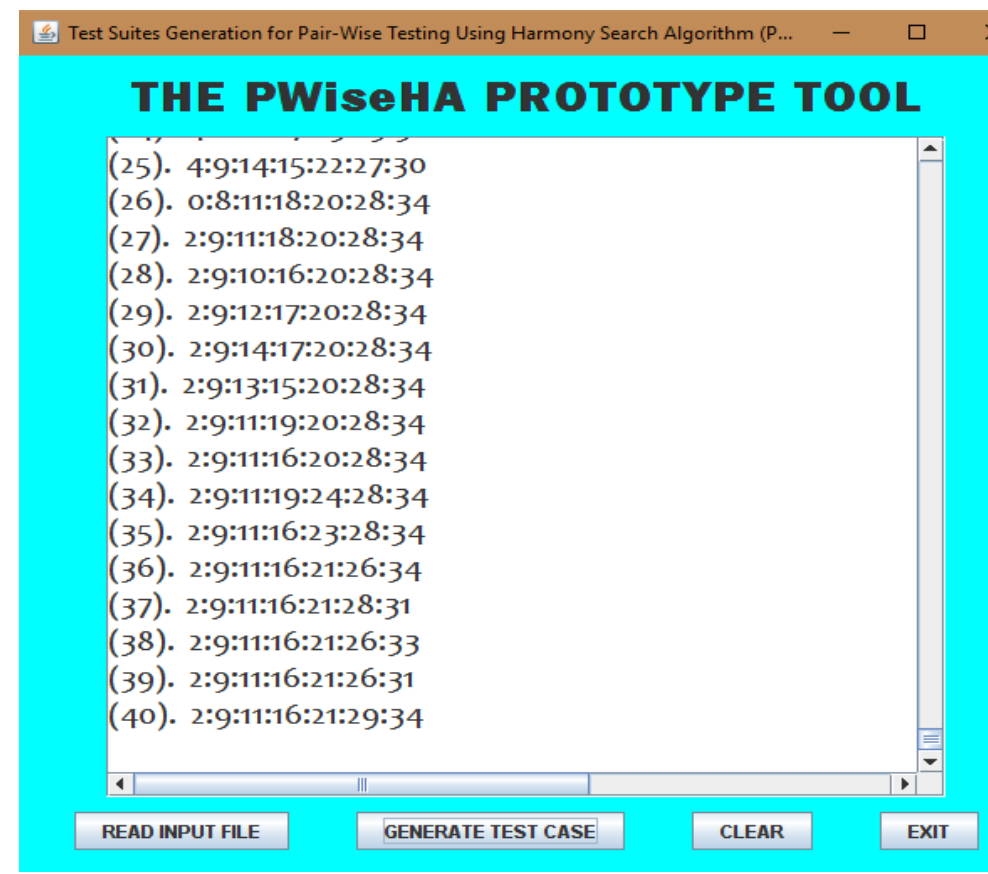

Figure 4. The PWiseHA form design with a displayed information of final test suite

\section{RESULTS AND DISCUSSION}

Evaluation of the PW is eHA mainly emphasized on the efficiency when generating a better final test suites sizes compared with existing strategies and the performance in terms of execution time when generating the final tes t suites.

We used the benchmark problems that are presented in pairwise testing website [17] to compare PWiseHA with those exis ting strategies. The website listed thirteen pairwise tools for generating final tes t suites, most of them with their efficiency measures [17]. These tools include: AETG, IPO, TConfig, CTS, Jenny, TestCover, DDA, AllPairs, PICT, EXACT, IPOS, ecFeed and JCUnit. The efficiencies of these tools were compared using six benchmark configurations with the notation $\mathrm{x}^{\mathrm{y}}$, where $\mathrm{x}$ represent the input parameters and $y$ represent their distinct values. The configurations appears on different sizes, which are as follows: $3^{4}, 3^{13}$, $4^{15} 3^{17} 2^{29}, 4^{1} 3^{39} 2^{35}, 2^{100}$, and $10^{20}$.

TABLE II: COMPARISON OF PWISEHA WITH SOME STRATEGIES IN [10] WHEN GENERATING BETTER AND BEST FINAL TEST SUITES

\begin{tabular}{|c|c|c|c|c|c|c|c|c|c|c|c|c|c|c|}
\hline نే & 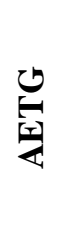 & ? & 苞 & $\underset{v}{U}$ & 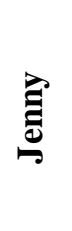 & 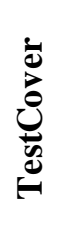 & $\widehat{\widehat{a}}$ & 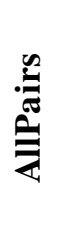 & $\underline{\underline{\theta}}$ & 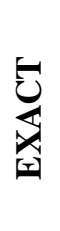 & $\stackrel{\mathscr{E}}{\varrho}$ & 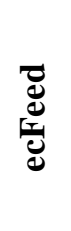 & 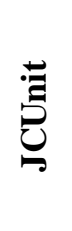 & $\begin{array}{l}3 \\
0 \\
0 \\
0 \\
0\end{array}$ \\
\hline $3^{4}$ & 9 & 9 & 9 & 9 & 11 & 9 & $?$ & 9 & 9 & 9 & 9 & 10 & 10 & 9 \\
\hline $3^{13}$ & 15 & 17 & 15 & 15 & 18 & 15 & 18 & 17 & 18 & 15 & 17 & 19 & 23 & 17 \\
\hline $4^{15} 3^{17} 2^{29}$ & 41 & 34 & 40 & 39 & 38 & 29 & 35 & 34 & 37 & $?$ & 32 & 37 & 49 & 44 \\
\hline $4^{1} 3^{39} 2^{35}$ & 28 & 26 & 30 & 29 & 28 & 21 & 27 & 26 & 27 & 21 & 23 & 28 & 33 & 27 \\
\hline $2^{100}$ & 10 & 15 & 14 & 10 & 16 & 15 & 15 & 14 & 15 & 10 & 10 & 16 & 18 & 22 \\
\hline $10^{20}$ & 180 & 212 & 231 & 210 & 193 & 181 & 201 & 197 & 210 & $?$ & 220 & 203 & 245 & 1048 \\
\hline
\end{tabular}


TABLE II displays the final test suite size of these tool, along side the PWiseHA, it displays equal or sometimes better efficiencies on all of the benchmark configurations except that of the last two configurations.

TABLE III: EVALUATION PERFORMANCE OF PWISEHA IN TERMS OF EXECUTING TIME WHEN GENERATING FINAL TEST SUITES.

\begin{tabular}{ll}
\hline Configuration & PWis eHA (Execution Time in sec) \\
\hline $\mathbf{3}^{\mathbf{4}}$ & 0.271 \\
\hline $\mathbf{3}^{\mathbf{1 3}}$ & 0.326 \\
\hline $\mathbf{4}^{\mathbf{1 5}} \mathbf{3}^{\mathbf{1 7}} \mathbf{2}^{\mathbf{2 9}}$ & 18.250 \\
\hline $\mathbf{4}^{\mathbf{1 3}} \mathbf{2}^{\mathbf{3 5}}$ & 9.232 \\
\hline $\mathbf{2}^{\mathbf{1 0 0}}$ & 3.810 \\
\hline $\mathbf{1 0}^{\mathbf{2 0}}$ & 45.600 \\
\hline
\end{tabular}

Performance in terms of execution time is also measured (but no comparis on), see TABLEIII for the evaluation which is also good, because almost each of the configurations is executed in less than a minute which means it can save time during execution which will be able to lead to saving of cost as well.

\section{CONCLUSION}

In this paper, we proposed and developed a strategy for pairwise tes ting called PWiseHA that generated an optimized final test suites by applying harmony search algorithm. Our evaluation results are inspiring, typically in terms of generating an optimal test suite in a suitable execution time. As part of our forthcoming work, PWiseHA is still in a prototype form, an obvious starting point for future work would be to complete theimplementation.

\section{REFERENCES}

[1] A. A. Muazu and A. A. Muazu. Design of a Harmony Search Algorithm Based on Covering Array T-Way Testing Strategy. $1^{\text {st }}$ International Conference on Information Technology in Education \& Development (ITED), ISBN: 978-978-35911-7-7, Page 33 - 38. April, 2018.

[2] X. Dianxiang, X. Weifeng, K. Michael, T. Lijo, and W. Linzhang. An Automated Test Generation Technique for Software Quality Assurance. IEEE transactions on reliability. VOL. 64, NO. 1. 2015.

[3] M. I. Younis, and K. Z. Zamli. A parallel t-way test generation strateg y for multicore systems. ETRI Journal. 32(1), 73-83. 2010.

\section{ACKNOWLEDGMENT}

This work is partly supported by a research grant of Tertiary Education TrustFund (TETFund) fromUmaru Musa Yar'adua University Katsina, Nigeria.
[4] A. B. Nasir, A. A. Alsewari, A A. Muazu and K. Z. Zamli. Comparative Performance Analysis of Flower Pollination Algorithm and Harmony Search based strategies: A Case Study of Applying Interaction Testing in the Real World. $2^{\text {nd }}$ International Conference on New Directions in Multidisciplinary Research \& Practice, ISBN: 978-969-9948-47-3, 2016.

[5] A. A. Alsewari and K. Z. Zamli. Design and implementation of a harmony-search-based variablestrength t-way testing strategy with constraints support. Journals on Information and Software Technology. 54, 553-568. 2012.

[6] F. Konrad, and L. Horst. Combinatorial Robustness Testing with Negative Test Cases. IEEE 19th International Conference on Software Quality, Reliability and Security (QRS). DOI 10.1109/QRS.2019.00018. 2019. 
[7] K. C. Ashis, C. Parna, C. Poulami and C. Aleena. Optimum Testing Time of Software using Size-Biased Concept. ArXiv: 1908.00307 v 1 [stat.ME] 1 Aug 2019.

[8] B. Hambling. Software testing an ISTQB-ISEB foundation guide. 2011.

[9] K. Tai, and Y. Lie. Test generation strategy using pairwise. IEEE transaction on software engineering. 28(1), 109-111. 2002.

[10] S. G. Laleh, C. Jaganmohan, L. Yu, K. Raghu, K. Richard BEN: a combinatorial testing-based fault localization tool. IEEE Eighth International Conference on Software Testing, Verification and Validation Workshops (ICSTW). 978-1-4799-1885-0. 2015.

[11] R. Bryce, and C. Colbourn. A density-based greedy algorithm for higher strength covering arrays. Software Testing, Verification and Reliability. 19(1), 37-53. 2009.

[12] J. Yan, and J. Zhang. Combinatorial testing: principles and methods. Journal of Software. 20(6), 1393-1405. 2009.

[13] K. Z. Zamli, M. Klaib, M. Younis, N. Is a and R. Abdullah. Design and implementation of a t-way test data generation strategy with automated execution tool support information science. Journal of information science. 181(9), 1741-1758. 2012.

[14] A. A. Alsewari and K. Z.Zamli. A harmony searchbased pairwise sampling strategy for combinatorial testing. International Journal of the Physical Sciences. 7(7), 1062 - 1072. 2012.

[15] Y. Cui, L. Li and S. Yao. A new strategy for pairwise test case generation. Third International Symposium on Intelligent Information Technology Application. 3, 303306. 2009.
[16] L. Z. Hasneeza, K. Z. Zamli. Migrating bird's optimization based strategies for pairwise testing. 9th Malaysian Software Engineering Conference. 978-14673-82274. 2015.

[17] J. Czerwonka (2019, oct.) Pairwise testing, combinatorial test case generation. [Online]. Available: http://www.pairwis e.org. 2019.

[18] A. Nahid and K. Susmita. Review Paper on Various Software Testing Techniques \& Strategies. Global Journal of Computer Science and Technology Volume XIX Issue II Version I. 2019.

[19] F. Pedro and C. Yoons ik. "PW iseGen: Generating Test Cas es for Pairwise Testing Using Genetic Algorithms". IEEE International Conference on Computer Science and Automation Engineering (CSAE 2011), Shanghai, China. 2011.

[20] A. A. Muazu and A. A. Muazu. One-Parameter-at-aTime combinatorial testing Strategy Based on Harmony Search Algorithm Supporting Mixed Covering Array Mathematical Notation (OPATHS). $1^{\text {st }}$ International Conference on Information Technology in Education \& Development (ITED), ISBN: 978 978-35911-7-7, Page 33 - 38. April, 2018.

[21] D. M. Cohen, S. R. Dalal, A. Kajla and G. C. Patton. The automatic efficient test generator (AETG) system Journals on International Symposium on Software Reliability Engineering (IEEEISSRE). 303-309. 1994.

[22] D. Manjarresa, I. Landa, S. Gil. A survey on applications of the Harmony search algorithm. Eng. Appl. Artif. Intel. 26(8), 1818-1831. 2013.

[23] Z. W. Geem, J. H. Kim, G. V. Li. A new heuristic optimization algorithm: Harmony search, Simulation 76 (2) (2001) 60-68. 2001.

[24] Z. W. Geem. (ed.), Music-Inspired Harmony Search Algorithm(Springer, Berlin, 2001). 2001. 